\title{
NATUREZA DO COQUE FORMADO SOBRE A MORDENITA DURANTE A TRANSALQUILAÇÃO DE BENZENO
}

Maria do Carmo Rangel*, Antoninho Valentini, Adriana Santana de Oliveira e Jorge Maurício David

Instituto de Química, Universidade Federal da Bahia, Campus Universitário de Ondina, 40170-290 Salvador - BA

Jaildes Marques Britto e Suzana Marques Domingues

Copene Petroquímica do Nordeste S.A., Rua Eteno, 1561, 42810-000 Camaçari - BA

Patrício Reyes

Facultad de Ciências Químicas, Universidad de Concepción, Casilla 3-C, Concepción - Chile

Recebido em 14/3/02; aceito em 26/8/02

\begin{abstract}
NATURE OF THE COKE FORMED ON MORDENITE DURING BENZENE TRANSALKYLATION. Zeolite catalysts have been extensively used in petroleum refining and the chemical industry although they are deactivated by coke deposition. In order to find the best condition to avoid deactivation, the coke formation on H-mordenite was studied in this work. The coke was produced during benzene transalkylation with $\mathrm{C}_{9}{ }^{+}$aromatics, under several reaction conditions. It was found that hydrogenated coke was produced in all samples without affecting the selectivity of toluene and xylene formation. This is explained in terms of the mordenite structure and the presence of hydrogen.
\end{abstract}

Keywords: mordenite; benzene transalkylation; coke.

\section{INTRODUÇÃO}

O interesse pelo estudo e pelo emprego de zeólitas como catalisadores tem aumentado de modo significativo, nas últimas décadas, devido à elevada acidez desses materiais e à sua peculiar estrutura de canais e cavidades. Entre os diversos aspectos estudados, os mais abordados são a seletividade dos catalisadores, sua resistência à desativação e seu envelhecimento ${ }^{1}$. A desativação é um dos problemas mais importantes, constituindo o principal obstáculo a muitas aplicações das zeólitas. Por essa razão, o tema tem sido amplamente estudado ${ }^{1-3}$.

O coque é, em geral, a principal causa de desativação das zeólitas, podendo envenenar os sítios ativos ou bloquear seu acesso ${ }^{4}$. Por outro lado, a remoção do coque pode degradar permanentemente os catalisadores, por desaluminização ou sinterização $0^{1,3}$. Dessa forma, procura-se desenvolver catalisadores que sejam resistentes ao coque ou estabelecer condições de reação que minimizem a sua formação.

É conhecido ${ }^{5}$ que a velocidade de formação do coque, sua composição e seu efeito desativante dependem da estrutura de poros das zeólitas, da acidez e das condições de operação, tais como temperatura, pressão e natureza dos reagentes. É de se esperar, por exemplo, que a mordenita seja rapidamente desativada devido à sua estrutura monodimensional ${ }^{6,7}$. A presença de uma molécula de coque num canal torna inacessíveis os sítios ativos a partir do local onde a molécula está localizada ${ }^{6,7}$.

Apesar de ser susceptível ao depósito de coque, a mordenita é amplamente utilizada em processos comerciais, sobretudo aqueles envolvendo hidrocarbonetos de alto peso molecular ${ }^{6-8}$. Nas reações de transalquilação de tolueno com cargas $\mathrm{C}_{9}$ (hidrocarbonetos com nove átomos de carbono), essas zeólitas também têm se apresentado como promissoras ${ }^{9}$. Entretanto, não existem trabalhos na literatura abordando a transalquilação do benzeno com cargas $\mathrm{C}_{9}$, bem como estudos da natureza do coque formado sobre a mordenita.

Considerando esses aspectos, neste trabalho estudou-se a natureza do coque formado sobre a mordenita durante a transalquilação

*e-mail: mcarmov@ufba.br de benzeno, com correntes ricas em compostos $\mathrm{C}_{9}{ }^{+}$(hidrocarbonetos com nove ou mais átomos de carbono), usando cargas de diferentes composições. Essas correntes são geradas como sub-produtos na reforma de nafta e no craqueamento de nafta, nos quais se formam quantidades apreciáveis de compostos aromáticos $\mathrm{C}_{7} \mathrm{e}_{9}{ }^{8-10}$. Essas reações oferecem a possibilidade de se desenvolver processos que não prejudiquem o meio ambiente, envolvendo custos de operação relativamente baixos ${ }^{10}$.

A transalquilação de tolueno com trimetilbenzenos é uma das reações de conversão de metilaromáticos mais estudadas, com a principal finalidade de produzir xilenos ${ }^{9}$. Entretanto, a transalquilação do benzeno com compostos aromáticos $\mathrm{C}_{9}{ }^{+}$surgiu, recentemente, como uma reação importante para aplicações comerciais e sua investigação tem sido cada vez mais intensa, principalmente por consumir benzeno, reconhecido agente cancerígeno ${ }^{8-10}$.

\section{PARTE EXPERIMENTAL}

Os catalisadores utilizados consistiram de pastilhas cilíndricas $(\phi=1,6 \mathrm{~mm}, l=5 \mathrm{~mm})$ de mordenita comercial, previamente secas sob nitrogênio a $350^{\circ} \mathrm{C}$, por $1 \mathrm{~h}$.

A reação de transalquilação de benzeno com uma corrente $\mathrm{C}_{9}^{+}$ foi conduzida num reator com agitação e controle automático de temperatura e pressão, em linha com um cromatógrafo a gás HP5890 provido de uma coluna Pona metil silicone de $50 \mathrm{~m}$. Os experimentos foram conduzidos a $30 \mathrm{~kg} \cdot \mathrm{cm}^{-2}, \mathrm{H}_{2} /$ hidrocarboneto $=8 \mathrm{e} \mathrm{WHSV}$ $=1 \mathrm{~h}^{-1}$, a diferentes temperaturas e usando diferentes correntes de alimentação. Estas correntes foram obtidas pela mistura de benzeno (99,8\% de pureza) e uma corrente $\mathrm{C}_{9}{ }^{+}$, numa razão benzeno/ $\mathrm{C}_{9}{ }^{+}=$ 70/30 (em peso).

Os testes catalíticos foram realizados sob condições que pudessem causar a desativação do catalisador. Dessa forma, cada amostra foi submetida a sucessivos testes catalíticos a diferentes temperaturas na faixa de 350 a $470{ }^{\circ} \mathrm{C}$. O seguinte procedimento foi empregado, com cada amostra: o catalisador foi aquecido a $350{ }^{\circ} \mathrm{C}$ sob hidrogênio, por $1 \mathrm{~h}$ e avaliado a $350{ }^{\circ} \mathrm{C}$, por $5 \mathrm{~h}$. Em seguida, foi aquecido a $380^{\circ} \mathrm{C}$, conduzindo-se a reação, nesta temperatura, por $5 \mathrm{~h}$. Este 
procedimento foi repetido nas temperaturas de 410,440 e $470{ }^{\circ} \mathrm{C}$, sucessivamente. Em cada temperatura determinava-se a composição da mistura reacional a intervalos de tempo de $70 \mathrm{~min}$. Foram monitoradas as concentrações de 72 hidrocarbonetos de $\mathrm{C}_{1}$ a $\mathrm{C}_{12}$, incluindo compostos parafínicos, olefínicos, naftênicos e aromáticos.

Foram conduzidas três séries de testes, usando-se três tipos de correntes: (i) com $2 \%$ de compostos aromáticos $\mathrm{C}_{10}{ }^{+}, 9 \%$ de propilbenzeno, $43 \%$ de etiltolueno, $45 \%$ de trimetilbenzeno e $0,6 \%$ de indano; (ii) com $10 \%$ de compostos aromáticos $\mathrm{C}_{10}{ }^{+}, 9 \%$ de propilbenzeno, $37 \%$ de etiltolueno, $44 \%$ de trimetilbenzeno e $0,6 \%$ de indano e (iii) com $20 \%$ de compostos aromáticos $\mathrm{C}_{10}{ }^{+}, 7 \%$ de propilbenzeno, $32 \%$ de etiltolueno, $40 \%$ de trimetilbenzeno e $1 \%$ de indano.

Após os testes catalíticos, os catalisadores foram caracterizados por análise química (teor de carbono), oxidação com temperatura programada acoplada à espectrometria de massas (TPO/MS) e análise térmica (DTA, TG).

O teor de carbono nos catalisadores foi determinado num aparelho Leco modelo CS-444, usando amostras previamente moídas em almofariz de ágata e peneiradas em 140 mesh.

As análises por TPO/MS foram conduzidas num equipamento Micromeritics modelo TPD/TPR 2900, acoplado a um quadrupolo de massas Hiden modelo Hal 200. As amostras foram secas a $120{ }^{\circ} \mathrm{C}$, durante a noite e, em seguida, novamente secas a $125^{\circ} \mathrm{C}$ por 30 min, sob argônio, imediatamente antes da análise. A seguir, foram resfriadas à temperatura ambiente e aquecidas numa velocidade de 15 graus/min sob fluxo de uma mistura $5,3 \% \mathrm{O} / \mathrm{He}(50 \mathrm{~mL} / \mathrm{min})$ até $862{ }^{\circ} \mathrm{C}$. Foram monitorados os seguintes sinais de massa $(\mathrm{m} / \mathrm{e})$ : $18\left(\mathrm{H}_{2} \mathrm{O}\right), 28(\mathrm{CO})$ e $44\left(\mathrm{CO}_{2}\right)$. O número total de mols de monóxido e dióxido de carbono foi medido pela quantidade de oxigênio consumido durante a queima do coque.

A análise térmica diferencial (DTA) foi realizada num instrumento Shimadzu modelo DTA50 a uma velocidade de aquecimento de 10 graus/min desde a temperatura ambiente até $1000^{\circ} \mathrm{C}$, sob fluxo de ar $(50 \mathrm{~mL} / \mathrm{min})$. Os experimentos de termogravimetria (TG) foram executados nas mesmas condições, usando um equipamento Shimadzu modelo TGA50.

A massa de coque das amostras foi extraída tratando-se o sólido com ácido fluorídrico $40 \%$, por $2 \mathrm{~h}$, à temperatura ambiente, seguido de refluxo com ácido clorídrico $36 \%$, por $2 \mathrm{~h}$. O coque foi separado por filtração. A fração solúvel do coque foi extraída mantendose as amostras, por $24 \mathrm{~h}$, num aparelho soxhlet, usando hexano e diclorometano p.a. seqüencialmente (nessa ordem). Empregou-se cerca de $300 \mathrm{~mL}$ de cada solvente e os extratos foram concentrados num evaporador rotativo. O coque solúvel foi, então, passado por uma coluna cromatográfica de sílica gel (60-230 mesh). O coque foi, em seguida, eluído com uma mistura hexano/acetato de etila (3:2) para purificar as amostras, que foram injetadas num cromatógrafo Hewlett Packard HP5790A acoplado a um espectrômetro de massas MS5970.

Os espectros no UV/VIS foram registrados utilizando-se um equipamento da Varian modelo Cary I, empregando-se soluções de $0,01 \mathrm{mg} / \mathrm{ml}$ de cada amostra em metanol grau espectrométrico. Os espectros foram registrados na faixa de 210-600 $\mathrm{nm}$ em cela de quartzo.

Os experimentos de FTIR foram conduzidos num equipamento Jasco modelo Valor-III usando amostras do coque solúvel (em suspensão de nujol) e do coque insolúvel (em pastilhas de brometo de potássio).

Os difratogramas de raios $\mathrm{X}$ da fração insolúvel do coque foram obtidos num equipamento Shimadzu modelo XRD-6000, usando radiação $\mathrm{CuK} \alpha$ gerada a $30 \mathrm{KV}$ e $30 \mathrm{~mA}$ e filtro de níquel.

\section{RESULTADOS E DISCUSSÃO}

A conversão dos compostos $\mathrm{C}_{9}{ }^{+}$e a seletividade a tolueno e xilenos, em função da temperatura, estão mostradas na Figura 1. Em todos os casos, a conversão aumentou com a temperatura. Após os testes conduzidos na temperatura mais alta $\left(470{ }^{\circ} \mathrm{C}\right)$, os catalisadores levaram à mesma conversão que aquela do primeiro teste $\left(350{ }^{\circ} \mathrm{C}\right)$, indicando que não ocorreu desativação. Pode-se também notar que o aumento na quantidade de compostos $\mathrm{C}_{10}{ }^{+}$na carga levou a um decréscimo na conversão total, em concordância com trabalhos anterio$\mathrm{res}^{8}$. Por outro lado, a seletividade a xilenos aumentou e a seletividade a tolueno diminuiu com o aumento do teor de hidrocarbonetos pesados.
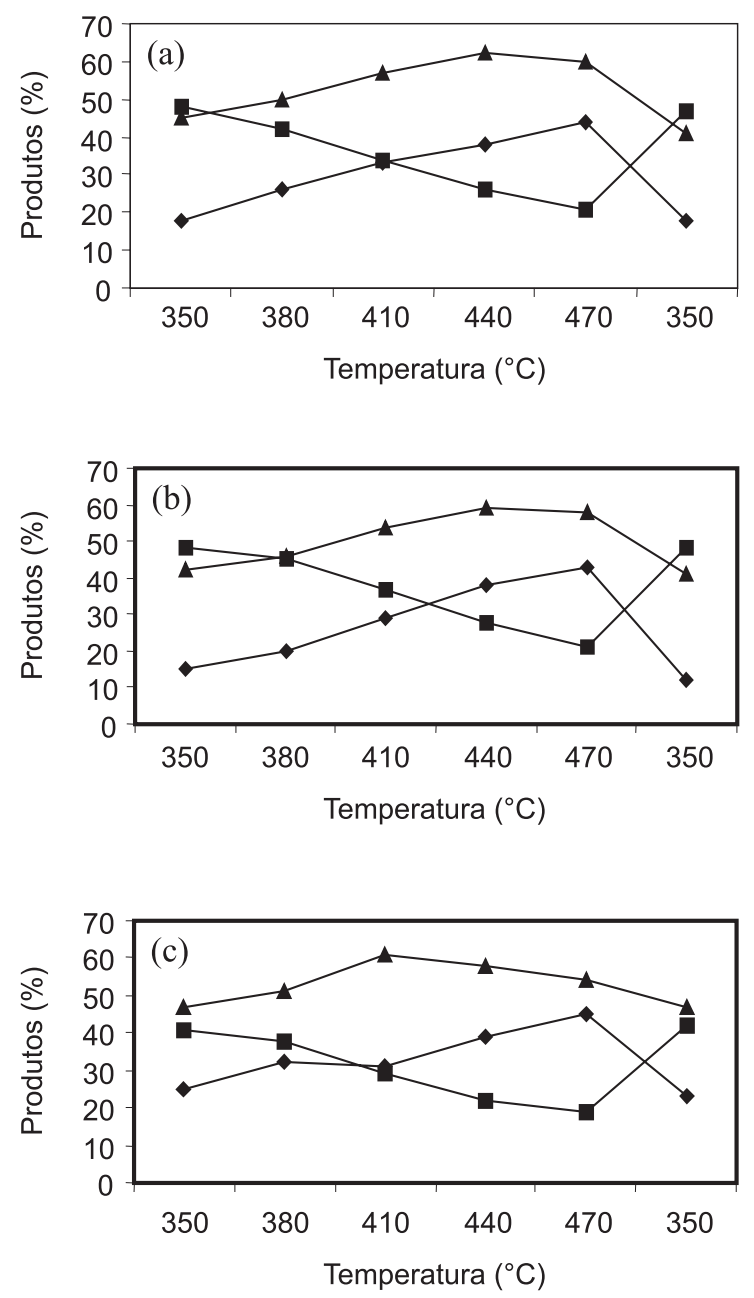

Figura 1. Efeito da temperatura sobre a formação dos produtos sobre (a) amostra M20 (coqueada com a carga $20 \% \mathrm{C}_{10}{ }^{+}$); (b) amostra M10 (coqueada com a carga $10 \% \mathrm{C}_{10}{ }^{+}$) e (c) amostra $\mathrm{M} 10$ (coqueada com a carga $10 \%$ $\left.\mathrm{C}_{10}{ }^{+}\right) \longrightarrow$ tolueno $\longrightarrow$ xilenos $\longrightarrow$ conversão total

Embora a conversão não tenha diminuído ao longo dos testes a diferentes temperaturas de reação, houve produção de coque em todas as amostras, como mostra a Tabela 1. As análises de carbono mostraram que os teores de carbono foram próximos entre si, sendo mais elevado no catalisador coqueado com a corrente mais rica em compostos $\mathrm{C}_{10}{ }^{+}$.

As curvas de TPO apresentaram dois picos a cerca de 186 e $527^{\circ} \mathrm{C}$ e não se notou diferença significativa entre os perfis das amos- 
Tabela 1. Teor de carbono (C), teor de coque solúvel relativo ao coque total (CS/CT) e teor de coque solúvel relativo ao catalisador (CS/CAT). Amostras M2, M10 e M20: coqueadas com cargas com $2 \%, 10 \%$ e $20 \%$ de compostos $\mathrm{C}_{10}{ }^{+}$, respectivamente

\begin{tabular}{lccc}
\hline Amostras & C (\%) & CS/CT $(\%)$ & CS/CAT $(\%)$ \\
\hline M2 & 3,1 & 11,2 & 0,4 \\
M10 & 2,8 & 10,8 & 0,3 \\
M20 & 3,6 & 36,2 & 0,6 \\
\hline
\end{tabular}

tras. A Figura 2 ilustra os termogramas obtidos. O primeiro efeito é devido à saída de água, como mostraram as análises por MS. O outro é devido à eliminação do monóxido e do dióxido de carbono e está relacionado à queima do coque. A baixa temperatura de queima mostra que o coque é hidrogenado. A razão molar $\mathrm{CO} / \mathrm{CO}_{2}$, por massa de catalisador, permaneceu na faixa de 1,0-2,2 x 10 $0^{-3}$, o que confirma a natureza hidrogenada do coque ${ }^{11}$.

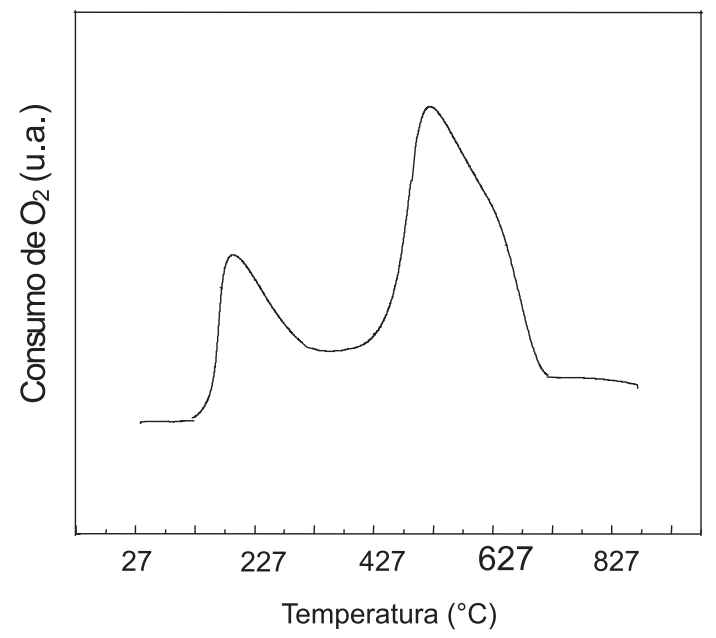

Figura 2. Termograma de TPO da amostra M20 (coqueada com carga com $20 \%$ de compostos $\mathrm{C}_{10}{ }^{+}$)

Os termogramas obtidos por DTA e TG são mostrados na Figura 3. Nota-se algumas diferenças nos perfis, como conseqüência da quantidade de compostos $\mathrm{C}_{10}{ }^{+}$na carga. Todas as curvas mostraram um pico endotérmico abaixo de $100{ }^{\circ} \mathrm{C}$, que pode ser atribuído à presença de água adsorvida e que também foi detectado com a mordenita nova. Pode-se, ainda, observar um pico a cerca de $550{ }^{\circ} \mathrm{C}$ nos perfis de DTA que é, provavelmente, devido à queima do coque mais hidrogenado localizado fora dos poros. Esse processo é acompanhado de perda de massa, como mostra a Figura 3(b). As curvas das amostras M10 e M20 mostraram um pico adicional a cerca de $640{ }^{\circ} \mathrm{C}$, que pode ser atribuído à queima do coque situado dentro dos poros. Este coque é provavelmente menos hidrogenado, uma vez que as moléculas dos precursores do coque têm pouco acesso ao hidrogênio, quando situadas no interior dos poros.

A partir dos experimentos de extração, observou-se que uma parte significativa do coque é solúvel. A amostra coqueada com a carga mais concentrada em hidrocarbonetos de alto peso molecular (Amostra M20) produziu a maior fração de coque solúvel, como mostrado na Tabela 1. Isto significa que, embora mais coque tenha sido produzido nesta amostra, grande parte dele é solúvel e, portanto, hidrogenado e fácil de ser removido.
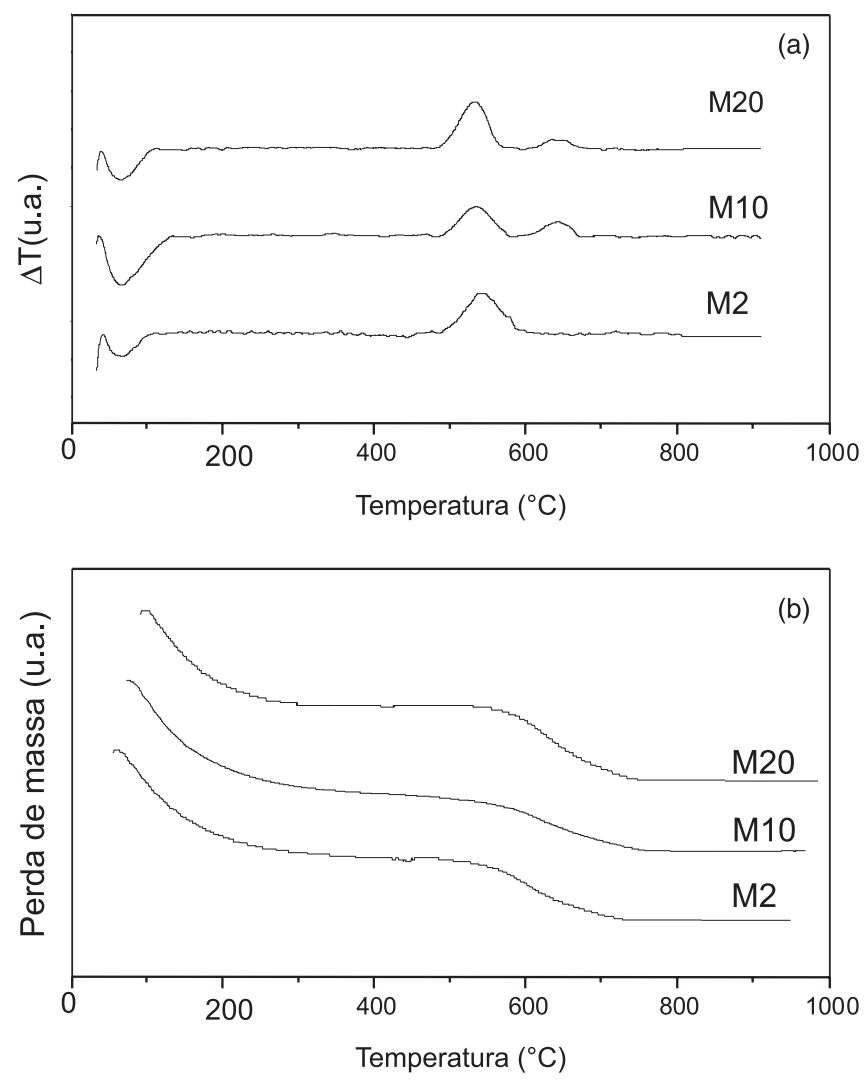

Figura 3. Termogramas de (a) DSC e (b) TG dos catalisadores coqueados. Amostras M2, M10 e M20: coqueadas com cargas com 2\%, 10\% e 20\% de compostos $\mathrm{C}_{10}{ }^{+}$respectivamente

Os espectros de FTIR da fração solúvel do coque mostraram bandas a 3010, 1480 e $780 \mathrm{~cm}^{-1}$ atribuídas às vibrações de estiramentos $\mathrm{C}-\mathrm{H}$ e C-C e às vibrações de deformação C-H e C-C, respectivamente, de compostos aromáticos ${ }^{12}$. Os espectros também mostraram bandas de absorção características de hidrocarbonetos alifáticos a 2960 e $2800 \mathrm{~cm}^{-1}$, referentes aos grupos $\mathrm{CH}_{3}$, e a $2930 \mathrm{~cm}^{-1}$, correspondente aos grupos $\mathrm{CH}_{2}{ }^{12}$. A banda atribuída ao coque, devido à presença de compostos poliolefínicos e/ou poliaromáticos, apareceu como um ombro a $1585 \mathrm{~cm}^{-13,14}$. Nenhuma diferença significativa foi observada entre os espectros de FTIR. A presença de compostos aromáticos foi confirmada por uma banda a $262 \mathrm{~nm}$ no espectro no UV $\mathrm{UV}^{15}$.

A análise por MS/GC indicou que as amostras de coque solúvel são constituídas por eicosano $\left(\mathrm{C}_{20} \mathrm{H}_{42}\right)$, docosano $\left(\mathrm{C}_{22} \mathrm{H}_{46}\right)$, tricosano $\left(\mathrm{C}_{23} \mathrm{H}_{48}\right)$, tetracosano $\left(\mathrm{C}_{24} \mathrm{H}_{50}\right)$, pentacosano $\left(\mathrm{C}_{25} \mathrm{H}_{52}\right)$, hexacosano $\left(\mathrm{C}_{26} \mathrm{H}_{54}\right)$, heptacosano $\left(\mathrm{C}_{27} \mathrm{H}_{56}\right)$, octacosano $\left(\mathrm{C}_{28} \mathrm{H}_{58}\right)$, nonacosano $\left(\mathrm{C}_{29} \mathrm{H}_{60}\right)$, triacontano $\left(\mathrm{C}_{30} \mathrm{H}_{62}\right)$ e hentriacontano $\left(\mathrm{C}_{31} \mathrm{H}_{64}\right)$. Não foi encontrado nenhum composto aromático através dessa técnica, o que pode ser atribuído ao limite de detecção do equipamento utilizado, aliado ao baixo teor desses compostos nos sólidos.

A distribuição desses compostos nos extratos mostra que o catalisador, coqueado por hidrocarbonetos mais leves (amostra M2), apresentou uma distribuição do tamanho de moléculas deslocada para valores de pesos moleculares mais altos. Assim, a maior parte das moléculas da fração solúvel da amostra M2 possui entre 35 e 40 átomos de carbono, enquanto na amostra M20 a maior parte das moléculas possui entre 25 e 30 átomos de carbono. A amostra M10 apresentou um comportamento similar ao da amostra M2.

Esses resultados estão em concordância com estudos anteriores conduzidos com a mordenita, na reação de desproporcionamento e 
transalquilação de tolueno, em que se observou que a fração solúvel do coque continha um baixo teor de compostos aromáticos, quando se formava pouca quantidade de coque (cerca de $2 \%)^{9}$. Entretanto, o coque solúvel tornava-se essencialmente não aromático quando quantidades maiores de coque eram formadas sobre o catalisador (cerca de $4 \%$ ). Isto foi atribuído ao fato das moléculas de coque poderem crescer apenas ao longo do comprimento dos canais da mordenita. Dessa forma, com o aumento do teor de carbono, o crescimento de cadeias lineares seria preferencial em relação à condensação de núcleos aromáticos.

Embora no presente trabalho a alimentação seja constituída principalmente por compostos aromáticos, é possível que o hidrogênio possa reagir com os carbocátions produzidos nos sítios ácidos, como resultado das reações de craqueamento, levando à formação de compostos alifáticos. Essa reação pode ocorrer mesmo na ausência de um sítio metálico, como observado em outros trabalhos ${ }^{8,16}$.

Os resultados também concordam com outros autores que encontraram, em outros catalisadores, que o coque formado na faixa de $300-450{ }^{\circ} \mathrm{C}$ é "leve", ou seja, formado por hidrocarbonetos de baixo peso molecular quando comparado ao "coque real", que foi definido como um material carbonáceo tratado a altas temperaturas e possuindo uma baixa razão $\mathrm{H}_{2}$ /hidrocarboneto ${ }^{17}$.

Os difratogramas de raios $\mathrm{X}$ da fração insolúvel do coque não mostraram picos devido ao coque grafitizado, o que confirma que o coque é hidrogenado ${ }^{18}$. Os espectros de FTIR também não mostraram a banda a $1620 \mathrm{~cm}^{-1}$, característica do coque com estrutura grafítica $^{19}$. Isto pode ser explicado considerando-se a quantidade de hidrogênio no sistema, assim como a alta pressão usada nos experimentos, em concordância com outros trabalhos abordando outros sistemas ${ }^{8,18}$.

A partir desses resultados, pode-se concluir que as três condições experimentais usadas na reação de transalquilação podem ser empregadas sem prejuízo para o desempenho do catalisador. Nessas condições, a mordenita apresenta cerca de $3 \%$ de coque, sem perda de atividade e seletividade. O coque produzido é principalmente leve, constituído por uma parte solúvel, com grandes quantidades de compostos alifáticos, e uma parte insolúvel, amorfa aos raios X.

De acordo com vários autores, a formação de coque em zeólitas é controlada pela estrutura dos poros e acidez e, portanto, a mordenita seria facilmente desativada ${ }^{9}$. Nossos resultados mostraram que, sob determinadas condições pode-se produzir coque leve (formado por hidrocarbonetos com baixo peso molecular e hidrogenado) e localizado principalmente fora dos poros, não prejudicando o catalisador.

Conclui-se, dessa forma, que o coque formado sobre o catalisador não afeta a sua estabilidade nas três condições empregadas (correntes com $2 \%, 10 \%$ ou com $20 \%$ de compostos $\mathrm{C}_{10}{ }^{+}$). Essas diferentes condições, por outro lado, conduzem a diferentes seletividades a tolueno e xilenos.

\section{CONCLUSÕES}

O coque depositado sobre a mordenita durante a transalquilação do benzeno com hidrocarbonetos $\mathrm{C}_{9}{ }^{+}$não desativa o catalisador, independentemente do teor de hidrocarbonetos $\mathrm{C}_{10}{ }^{+}$na mistura reagente. Isto foi atribuído à natureza e à localização do coque: nas condições experimentais empregadas $\left(\mathrm{P}=30 \mathrm{~kg} . \mathrm{cm}^{-2}, \mathrm{H}_{2} /\right.$ hidrocarboneto $=8 \mathrm{e}$ WHSV $=1 \mathrm{~h}^{-1}$ ), o coque produzido é hidrogenado e constituído por hidrocarbonetos com baixo peso molecular, estando situado principalmente fora dos poros do catalisador. Isto é conseqüência da estrutura da mordenita, da presença de hidrogênio e da pressão usada. A quantidade de compostos $\mathrm{C}_{10}{ }^{+}$na corrente de reagente (com 2\%, $10 \%$ ou $20 \%$ de compostos $\mathrm{C}_{10}{ }^{+}$) não influencia na desativação do catalisador, mas conduz à produção de diferentes quantidades de tolueno e xilenos.

\section{AGRADECIMENTOS}

Os autores agradecem o suporte financeiro do PADCT/FINEP e CNPq. A. S. Oliveira e A. Valentini agradecem as bolsas de estudos. Os autores também agradecem a ajuda de M. L. Corrêa nos experimentos de difração de raios $\mathrm{X}$.

\section{REFERÊNCIAS}

1. Bhatia, S.; Beltramini, J.; Do, D. D.; Catal. Rev. -Sci. Eng. 1989, 31, 431.

2. Guisnet, M.; Magnoux, P.; Cartraud, P.; Mignard, S.; J. Catal. 1987, 106, 242.

3. Guisnet, M.; Magnoux, P.; Catal. Today 1997, 36, 477.

4. Guisnet, M.; Magnoux, P. Em Studies in Surface Science and Catalysis; Delmon, B.; Froment, G., eds.; Elsevier: Amsterdam, 1994.

5. Fouche, V.; Magnoux, P.; Guisnet, P.; Appl. Catal. 1990, 58, 189.

6. Das, J.; Bhat, Y. S.; Halgeri, A.; Catal. Lett. 1994, 23, 161

7. Guisnet, M.; Magnoux, P.; Appl. Catal. 1989, 59, 1.

8. Tsai, T.; Liu, S.; Wang, I.; Appl. Catal., A 1999, 181, 355.

9. Wu, J. C.; Leu, L. J.; Appl. Catal. 1983, 7, 283.

10. Forni, G.; Cremona, F.; Missineo, G.; Bellusi, C.; Perego, C.; Pazzuconi, G.; Appl. Catal., A 1995, 121, 261.

11. Choudhary, V. R.; Sivadinarayana, C.; Devadas, P.; Sansare, S. D.; Magnoux, P.; Guisnet, M.; Microporous Mesoporous Mater. 1998, 21, 91.

12. Silverstein, R.; Bassler, G. C.; Morrill, T. C.; Spectrometric Identification of Organic Compounds, John Wiley and Sons: New York, 1979, p. 121.

13. Karge, H. G.; Boldingh, E. P.; Catal. Today 1988, 3, 53.

14. Karge, H. G.; Boldingh, E. P.; Catal. Today 1988, 3, 379

15. Flego, C.; Pazzuconi, G.; Bencini, E.; Perego, C. Em Studies in Surface Science and Catalysis, Delmon, B.; Froment, G. F., eds.; Elsevier: Amsterdam, 1999.

16. Grau, J.; Parera, J. M.; Actas de $12^{\circ}$ Simposio Iberoamericano de Catalisis, Rio de Janeiro, Brasil, 1990

17. Radwan, A. M.; Kyotani, T.; Tomita, A.; Appl. Catal., A 2000, 192, 43.

18. Barbier, J. Em Studies in Surface Science and Catalysis; Delmon, B.; Froment, G.; eds.; Elsevier: Amsterdam, 1987.

19. Eisenbach, D.; Gallei, E. ; J. Catal. 1979, 56, 377. 